\title{
Flavonoids: Antioxidant Compounds for Plant Defence... and for a Healthy Human Diet
}

\author{
Oscar VICENTE ${ }^{1 *}$, Monica BOSCAIU ${ }^{2}$ \\ ${ }^{1}$ Universitat Politècnica de València, Instituto de Biologia Molecular y Celular de Plantas (IBMCP, UPV-CSIC), 46022 Valencia, \\ Spain; ovicente@ibmcp.upv.es (" correspondingauthor); \\ ${ }^{2}$ Universitat Politècnica de València, Instituto Agroforestal Mediterráneo (IAM, UPV), 46022 Valencia, \\ Spain;mobosnea@eaf.upv.es
}

\begin{abstract}
Flavonoids are a large group of plant phenolics, including almost 10,000 different compounds with a common chemical structure consisting of two aromatic rings, joined by a three-carbon chain generally forming a heterocyclic ring (C6-C3-C6). Interest on these secondary metabolites has increased exponentially in the last years, for its alleged beneficial effects on human health. It has been reported that flavonoids - and other phenolics - show antibacterial, antiviral, anti-inflammatory, antilipidemic, or antidiabetic activities, and also possess neuroprotective, hepatoprotective or cardioprotective properties. Anthocyanins, particularly, seem to be effective antitumoural compounds, at least in human tumour cell lines and in mouse models. These properties appear to be due to the strong antioxidant character of flavonoids and their capacity to scavenge 'reactive oxygen species' (ROS) which, if in excess, cause oxidative cellular damage. Therefore, flavonoid-rich fruits and vegetables should contribute to a healthy diet. Yet flavonoids are not present in plants for human benefit. They fulfil many disparate biological functions, mostly mediating interactions between plants and the environment: animal attractants for pollination and seed dispersal, signalling molecules in plant-microorganisms interactions, or participating in plant defence against pathogens. They are also involved in the mechanisms of tolerance to practically all types of abiotic stress, including UV radiation, extreme temperatures, ozone exposure, drought or salinity. Since abiotic stresses cause an increase in cellular ROS levels, these latter functions appear to be based on flavonoids' antioxidant activity, similarly to their assumed positive effects for human health if used as dietary components, nutraceuticals or even pharmacological drugs.
\end{abstract}

Keywords: antioxidants, phenolic compounds, ROS, abiotic stress responses

\section{Introduction}

Flavonoids and other phenolic antioxidants

Flavonoids are polyphenolic compounds synthesised by plants, with a basic structure (C6 - C3 - C6), composed of two aromatic rings joined by a three carbon chain, typically organised as an oxygenated heterocycle. Largely depending on the degree of oxidation of the heterocycle, flavonoids can be divided in turn into distinct subfamilies: chalcones, flavonols, flavanols, flavanones, flavones, isoflavones, anthocyanidins and their glycosides, condensed tannins, etc. The number and position of hydroxyl groups on the aromatic rings define the various individual compounds within each subfamily. The number of possible combinations of these substituent groups is huge, and more than 9,000 different flavonoids have been identified in plants so far (Williams and Grayer, 2004).
Flavonoids are only a subgroup of the even larger family of phenolic compounds, which also include monophenols (simple phenols and phenolic acids, such as hydroxybenzoic and hydroxycinnamic acids), coumarins and other polyphenols: tannins, lignins, lignans or stilbenes. Phenolics represent the most complex group of the so-called 'secondary metabolites' of plants, which collectively synthesise tens of thousands of different chemical structures, with a wide range of biological functions, characterised by containing hydroxylated aromatic rings.

The presence of multiple hydroxyl groups in their structures gives flavonoids and other phenolics a reducing character. In fact, it has been shown in in vitro assays that many of these compounds possess a strong antioxidant activity. This activity is particularly high, three to fourfold higher than in other flavonoids, in ortho-dihydroxy flavonoids - those containing a catechol group in their aromatic rings, such as flavonols or flavanols (Rice-Evans et al., 1996). Nitration of those catechol groups with $\mathrm{NaNO}_{2}$, followed by reaction with $\mathrm{AlCl}_{3}$, is the basis of a simple 
spectrophotometric assay to quantify 'antioxidant flavonoids' in plant extracts (Zhishen et al., 1999) although other phenolics bearing this chemical group (e.g. caffeic acid) are also detected (Pękal and Pyrzyska, 2014). This antioxidant activity seems to be the basis of many of their biological functions, being able to inactivate 'reactive oxygen species' (ROS). ROS are chemically reactive molecules that include, among others, free radicals such as superoxide $\left(\mathrm{O}_{2}{ }^{*}\right)$, hydroxyl $\left(\mathrm{OH}^{*}\right)$ and perhydroxyl $\left(\mathrm{O}_{2} \mathrm{H}^{*}\right)$ radicals, and other oxidant molecules such as singlet oxygen $\left({ }^{1} \mathrm{O}_{2}\right)$, molecular oxygen $\left(\mathrm{O}_{2}\right)$, ozone $\left(\mathrm{O}_{3}\right)$ or hydrogen peroxide $\left(\mathrm{H}_{2} \mathrm{O}_{2}\right)$ (Takahashi and Asada, 1988; Apel and Hirt, 2004). ROS are by-products of important metabolic processes and are normally present at low concentrations in the cell, where they fulfil some essential functions, for example as signalling molecules controlling cell growth and differentiation and stress responses (Pollastri and Tattini, 2011). Yet ROS levels can increase dramatically under different stress conditions, causing significant cellular damage. ROS affect the stability and permeability of cell membranes by lipid peroxidation, can inactivate proteins by oxidation of amino acid residues, and produce mutations by reaction with the bases of DNA (Halliwell, 2006).

The study of the molecular mechanisms that mediate the multiple biological functions of flavonoids in plants is a matter of indisputable academic interest. However, research on these compounds has increased greatly in recent years, not due to that basic research interest but rather to their alleged beneficial effects on human health. Flavonoids, especially, but also other phenolic compounds, have been shown to possess a wide range of biochemical and pharmacological activities, including antibacterial, antiviral, anti-inflammatory, antilipidemic, antidiabetic, neuroprotective, hepatoprotective and cardioprotective properties, and it has been suggested that they can be used as health-promoting, disease-preventing dietary supplements (Middleton et al., 2000; Coman et al., 2012; Kumar and Pandey, 2013; Ravishankar et al., 2013; Romano et al., 2013).

\section{The 'lipid hypothesis' and the 'French paradox'}

Nowadays, the role of cholesterol in the pathogenesis of atherosclerosis and cardiovascular disease is well established in medicine. This is largely due to the pioneering epidemiological studies of Ancel Keys, which allowed him to propose the 'lipid hypothesis', highlighting the relationship between the fat content of the diet, the level of total blood cholesterol and the incidence and mortality of cardiovascular diseases. After a series of tests on a smaller scale, in 1956 Keys examined the dietary habits of middleaged men of Japanese ancestry in their native Japan and in Hawaii and Los Angeles; despite the common genetic origin, the incidence of myocardial infarction was shown to be much higher in these latter two populations ( 4 to 1 and 10 to 1 , respectively) with respect to the native Japanese. While Japan's population received only $13 \%$ of their calories from dietary fat, this value increased to $32 \%$ for Japanese in Hawaii and to $45 \%$ in Los Angeles. Mean levels of total cholesterol corresponded to the three dietary patterns (Keys and Grande, 1957). These data were corroborated in a larger study, begun in 1957, which lasted several years and would eventually be known as the 'Study of the Seven Nations', involving 12,000 men 40-59 years old in the United States, Japan, Netherlands, Finland, Yugoslavia, Italy and the Greek Islands. In this study, Keys and his colleagues found that in societies where fat is a major component of the diet (USA and Finland) levels of blood cholesterol and the incidence of death from coronary heart disease were highest; by contrast, in countries of the Mediterranean region, with a diet based on fresh fruits, vegetables, bread, pasta and olive oil, dietary cholesterol in blood and the proportion of deaths from heart attacks were lower (Keys, 1970).

Initially, Keys' studies were harshly criticised, for alleged methodological flaws and for not including data from other countries that were available but seemed to go against the 'lipid hypothesis' (Yerushalmy and Hilleboe, 1957). However, many later works confirmed and extended Keys' findings, showing that the correlation with the incidence of coronary heart disease was more significant if saturated animal fat in the diet, rather than total fat, was considered; moreover, a negative correlation was even observed between cardiovascular disease incidence and diets rich in unsaturated vegetable oils or omega-3 fatty acids. Nevertheless, the general trend does not seem to apply to some countries, for example in France, where consumption of saturated fat in the diet is similar to other Central European countries, yet the incidence of cardiovascular disease and deaths from heart attack infarction are significantly lower. This is known as the 'French paradox' (St Leger et al., 1979), and has been attributed to consumption by the French of higher amounts of red wine, which contains relatively high levels of 'healthy' phenolic compounds such as flavonoids and resveratrol.

\section{Resveratrol: the wonderful molecule}

Resveratrol is a phenolic compound belonging to the group of stilbenes, which have a structure C6 - C2 - C6 (two aromatic rings joined by a chain of two carbons), thus very similar to that of flavonoids. Resveratrol was discovered in 1940 and is present in some berries, peanuts, walnuts and some other vegetables, as well as in the skin of grapes and in red wines. Resveratrol attracted little attention until 1992 when it was proposed as responsible for the cardioprotective effects of red wine, in which it is present in relatively large amounts; in fact, red wine constitutes the main source of resveratrol in the diet (Siemann and Creasy, 1992). This idea was supported by subsequent studies. Therefore, resveratrol would explain the 'French Paradox' (see Kopp, 1998; Vidavalur et al., 2006, for reviews), possibly in combination with flavonoids and other wine phenols.

Studies on the therapeutic potential of resveratrol increased exponentially following a seminal publication by Jang et al. (1997), which provided strong evidence of the cancer chemopreventive activity of this molecule, which inhibited a number of cellular events associated with the initiation, promotion and progression of tumours. These anticancer effects were due, apparently, to resveratrol antioxidant, anti-inflammatory and antimutagenic activities, as it was shown in various in vitro assays and cell 
16

culture systems. What was most interesting was that resveratrol also inhibited tumour formation in vivo, in a mouse skin cancer model. Further preclinical studies, both in vitro and in vivo, confirmed the chemoprotective and chemotherapeutic activities of this molecule, both regarding its anticancer effects, and its protective activity against pathological inflammation, viral infections, diabetes and cardiovascular diseases, myocardial infarction, stroke and brain damage (see Baur and Sinclair, 2006; Athar et al., 2007, for reviews).

As if the beneficial effects mentioned so far were not enough, resveratrol has also been defined as 'the molecule of eternal youth', after data were published demonstrating that it slows down the aging process and can prolong lifespan (both average and maximum), at least in yeast (Howitz et al., 2003), invertebrates like the worm Caenorhabditis elegans and the fly Drosophila melanogaster (Wood et al., 2004) or even vertebrates such as the short-lived fish Nothobranchius furzeri (Valenzano et al., 2006). It is assumed that this effect of resveratrol is mediated by its activity as a potent inducer of sirtuins, a protein family conserved in evolution, whose main function seems to be promoting the survival and resistance to stress in times of adversity, and whose overexpression increases lifespan in diverse organisms (Koubová and Guarente 2003; Guarente and Picard 2005).

\section{Beyond resveratrol: Flavonoids and other phenolic compounds}

For all the aforementioned reasons, research on the beneficial health effects of resveratrol has aroused great interest in recent years. However, many other studies in vitro, in cell cultures or in animal models have shown that other phenolic compounds, especially flavonoids, have similar protective activities against inflammatory processes, cardiovascular diseases or cancer (e.g. Middleton et al., 2000). Thus, in a model of chemically-induced oral carcinogenesis in hamsters, a lower incidence of tumours in the animals was observed in the presence of various phenolic compounds; apigenin (a flavone) and rosmarinic acid (a caffeic acid ester) were the most effective of the tested molecules (Baldasquin-Caceres et al., 2014). Anthocyanins also appear to be metabolites with anticancer activity. Using $\operatorname{Trp} 53^{-/}$mice, highly susceptible to tumour formation, it was found that inclusion in the diet of transgenic tomatoes which had a high content of anthocyanins (giving them a strong purple colour), by overexpression of two appropriate transcription factors, caused a significant extension of the lifespan of the mice, with respect to controls with their diet supplemented with normal tomatoes (Butelli et al., 2008). Skin extracts of a different anthocyanin-rich, purple tomato variety - in this case, obtained by conventional breeding also inhibited cell proliferation of two human tumour cell lines in culture (Mazzucato et al., 2013). The effect of lifespan increase is also not specific for resveratrol, as it has been shown as well for other antioxidant polyphenols. For example, the use of culture medium supplemented with a cocoa powder with high flavonoid content led to increased resistance to oxidative stress and to increased lifespan in $C$. elegans; these effects were fully dependent on the polyphenols present in the cocoa powder and on SIR-2.1, a worm sirtuin (Martorell et al., 2011).

\section{Enter 'snake oil salesmen': Limitations of phenolics as}

\section{dietary supplements}

All these data have promoted a huge commercial supply of these phenolic compounds, particularly resveratrol and flavonoids, to be used directly as food or food supplements, as nutraceutical or 'natural' compounds with pharmacological activity, or as cosmetic supplements. These products are generally crude or partially purified plant extracts, more or less concentrated, presented in multiple forms: capsules, tablets, gels, liquid solutions, etc., and sold at quite expensive prices. Advertising, allegedly supported by scientific studies, attributes them all kinds of benefits to health and wellness, and proposes their use in a wide range of applications: as anti-aging agents, for skin care, to stimulate the immune system, for weight loss, as anti-fatigue treatments, to eliminate tinnitus ('ringing in the ears') ... Of course, an excellent business but, as of today, without a sound scientific basis.

This does not mean that some of these compounds, or derivatives thereof, may not become effective medications for the treatment of certain diseases. Moreover, inclusion in the diet of plant foods rich in these antioxidant compounds may not be the solution to all problems mentioned above, but cannot be unhealthy - a notion that is strongly supported by epidemiological and statistical studies. However, for a full demonstration of the pharmacological and nutraceutical activities of specific flavonoids and other phenolics many studies are still needed since research on the effects on human health of these secondary metabolites presents many unresolved issues:

a) Despite the large number of studies in vitro, in cell systems and in animal models, there are not yet, to our knowledge, completed standard clinical trials of resveratrol and other polyphenols on which to base their supposedly beneficial effect on humans.

b) One of the biological functions of many of these compounds in plants is the defence against pathogens and herbivores (see below), for which they are toxic. This can also apply to humans at concentrations that are pharmacologically active. In fact, at least one initiated clinical trial had to be suspended due to the toxicity of the tested compound.

c) Concentrations of these compounds in fruits, vegetables or red wine (as in most commercial products sold as dietary supplements) are generally much lower than those that have proven effective in vitro or in animal models. For example, to achieve resveratrol levels equivalent to those used in experiments with mice, it would be necessary to drink more than 1000 litres of red wine per day!

d) The bioavailability of these compounds in the body may be very limited, due to low absorption in the intestine after oral ingestion and rapid degradation in the body (short half-life). Furthermore, metabolism of phenolics leads to the formation of side products, different in each case, the activity and effects of which are generally unknown. 
e) The conditions of the experiments in vitro may be incompatible with the conditions in the body. For example, many phenolic compounds are insoluble in water at neutral or acidic $\mathrm{pH}$, so that experiments are generally performed at alkaline $\mathrm{pH}$ - which can affect the antioxidant activity of the compounds - or in the presence of high concentrations of ethanol.

Nevertheless, in some of the experiments with animal models described in the previous paragraphs, significant positive results were obtained just by supplementing the animals' diet with flavonoid-rich plants or plant extracts (not with high doses of the purified compounds). This suggests that, at least in some specific cases, claims on the effective use of flavonoids as food supplements and nutraceuticals - not to mention as therapeutical drugs may turn out to be true, highlighting the interest of pursuing these studies.

\section{Biological functions of flavonoids (and other phenolics) in plants}

Obviously, phenolic compounds are not present in plants for the benefit of human consumers. These secondary metabolites play a wide range of biological functions in plants (Treutter, 2005, 2006; Gould and Lister, 2006; Pollastri and Tattini, 2011). Some phenolics such as lignin or hydroxycinnamic acids are structural components of the cell wall. Flavonoids are involved in various processes of growth and development, in some of them through the inhibition of polar auxin transport; this has been confirmed using Arabidopsis transparent testa mutants, which carry a mutation in the gene encoding chalcone synthase, the first enzyme of the flavonoid biosynthetic pathway (Brown $e t$ al., 2001). Moreover, a specific subgroup of flavonoids, the flavonols (quercetin, kaempferol, myricetin) can function as plant hormones, stimulating the formation of functional pollen. This has been shown using an in vitro system for tobacco pollen maturation in isolated microspore cultures (Ylstra et al., 1992), and also natural mutants affected in flavonoid synthesis, for example in petunia, which can be chemically or genetically complemented with flavonols (Taylor and Jorgensen, 1992; Napoli et al., 1999). However, in most cases, flavonoids are involved in the interactions of plants with their environment, as described in the following sections.

\section{Role of flavonoids in the interactions of plants with other living organisms}

Flavonoids are the major pigments responsible for the coloration of pollen and flowers (in the latter case, basically, anthocyanins); together with other compounds such as carotenoids, they also contribute to fruit colour. Thus, apart from the direct effect on pollen gametophytic development mentioned above, these compounds play an important role in plant reproduction, as attractants of pollinators and of animals responsible for the dispersion of fruits and seeds.

Flavonoids also act as signalling molecules in interactions between plants and microorganisms (Harborne and Williams, 2000; Treutter, 2005, 2006; Gould and
Lister, 2006; Cheynier et al., 2013). They are involved in the symbiosis between legumes and nitrogen-fixing bacteria, specifically in the process of legume nodulation: flavonoids, secreted by legume roots, activate transcription of nod (nodulation) genes in rhizobia, by binding to bacterial NodD transcription factors, thus triggering the expression of the proteins responsible for the synthesis of Nod factors, which activate nodule development and the first steps of infection. Different legumes produce distinct flavonoids, which bind to NodD transcription factors of particular rhizobia species, thus contributing to the specificity of the interaction (Downie, 2014). These compounds also participate in the mechanisms of plant defence against herbivores and pathogens: fungi, viruses and bacteria. Many flavonoids - as well as resveratrol and other phenols - are phytoalexins, i.e. antimicrobial compounds toxic to a wide range of pathogenic bacteria and fungi; their synthesis is rapidly induced in some plants after infection, so that high concentrations accumulate in a relatively short period of time, helping to limit the spread of the pathogen (Treutter, 2006). Moreover, phenolic compounds play an essential role in the infection of plants by Agrobacterium tumefaciens, the causative agent of crown gall disease. Wounded plant cells produce and secret acetosyringone (AS) or similar compounds (non-flavonoid phenolics), which attract the bacteria to the wound sites by chemotaxis and induce specifically the expression of the vir genes in the bacterial Ti plasmid. The proteins encoded by the virA and $v i r \mathrm{G} 2$ genes constitute a two-component sensor/regulator system that binds AS, activating the transcription of the other vir genes, necessary for the transfer of T-DNA to plant cells (Pacurar et al., 2011)

\section{Flavonoids in plant defence mechanisms against abiotic} stress

There is plenty of evidence that flavonoids and other phenolics are also involved in the responses of plants to many different types of abiotic stress, such as UV radiation and intense visible light, cold and high temperatures, exposure to ozone or heavy metals, anoxia, mineral nutrient imbalance, herbicides, drought or soil salinity (WinkelShirley, 2002; Treutter, 2005, 2006; Gould and Lister, 2006; Pollastri and Tattini, 2011; Di Ferdinando et al., 2012; and references therein).

\section{Protection against $U V$ light}

Several lines of evidence support the role of flavonoids in plant protection against UV light, which was first demonstrated in studies with Arabidopsis mutants: mutants affected in the flavonoid biosynthesis pathway were shown to be extremely sensitive to UV radiation ( $\mathrm{Li}$ et al., 1993); furthermore, high constitutive levels of flavonoids and other phenolics were detected in a mutant tolerant to lethal doses of UV-B light (Bieza and Lois, 2001). There are also some examples of a good positive correlation between UV exposure and flavonoid accumulation in plants (e.g., Stapleton and Walbot, 1994; Lavola, 1998; Jaakola et al., 2004). Furthermore, increased flavonoid levels have been measured at higher altitudes in several plant species 
(Bachereau et al., 1998; Zidorn et al., 2005; Rieger et al., 2008; Spitaler et al., 2008; Murai et al., 2009). Higher altitude means a higher intensity of solar UV radiation since less is filtered by the atmosphere (Blumthaler $e t$ al., 1997); therefore these results also point to the accumulation of flavonoids, at least in part, in response to UV stress. Indeed, the molecular mechanism underlying this response is well known: the expression of chalcone synthase, the first enzyme in the flavonoid biosynthesis pathway, is transcriptionally activated by UV light, as it has been demonstrated in several plant species (e.g. Koes et al., 1989; Schulze-Lefert et al., 1989).

Concerning the mechanisms of plant protection against UV radiation, the possible effect of polyphenols as direct UV screens has been regarded by several authors as a key biological function of these compounds (Rozema et al., 1997, 2002; Burchard et al., 2000). It has even been suggested that development of the biosynthetic pathways of phenolic compounds has been essential in the evolution of terrestrial plants, as lignins and flavonoids are lacking in almost all algae groups. More recent data have challenged this hypothesis, indicating that direct screening of UV light is not the most important mechanism of photoprotection mediated by flavonoids, which predominantly act scavenging UV-induced ROS; that is, as antioxidant compounds (Pollastri and Tattini, 2011; Agati et al., 2013).

The role of flavonoids in the defence against other abiotic stresses

Not only UV radiation, but many other environmental stress conditions cause oxidative stress in plants, either directly or as a secondary effect. Therefore, a general response of plants to abiotic stress is based on the activation of antioxidant systems, both enzymatic and non-enzymatic; many flavonoids and other phenolics can be included in the latter group (Apel and Hirt, 2004). Antioxidant flavonoids can be considered as a second line of defence against oxidative stress, which is activated only if the primary response - increase in antioxidant enzyme activities - is not efficient enough to eliminate excess ROS; therefore, flavonoid biosynthesis is predominantly activated under severe stress conditions, when the activities of antioxidant enzymes decline (Fini et al., 2011).

\section{Water and salt stress}

Drought and soil salinity are the environmental stress conditions that cause the most important losses of agricultural production worldwide, a problem that will worsen in the coming decades due to the forecasted effects of climate change (Fita et al., 2015); this has prompted intensive research on the mechanisms of plant defence against these stresses. Focusing on the activation of nonenzymatic antioxidant systems, there are several reports describing an increase in flavonoid contents in plants subjected to water deficit stress. For example, transcriptomic and metabolomic approaches comparing wild-type and Arabidopsis thaliana mutants, have revealed that flavonoid accumulation is essential to improve drought tolerance in this model species (Nakabayashi et al., 2014). In white clover, water deficit also increased significantly quercetin (a flavonol) contents, which were higher in the more droughtresistant genotypes (Ballizany et al., 2012). Flavonols have also been shown to increase under water stress conditions in other species, such as Crataegus laevigata and C. monogyna (Kirakosyan et al., 2003), whereas flavonoids and hydroxycinnamates - also efficient in vitro ROS scavengers - are involved in the responses Ligustrum vulgare to drought and to high solar radiation (Tattini et al., 2004). Similarly, an increase in flavanol levels has been reported in Cistus clusii plants upon controlled drought treatments, and in plants collected from the field in summer, characterised by high temperatures and prolonged lack of rain in the Mediterranean climate (Hernández et al., 2004).

There are fewer reports on the accumulation of flavonoids in response to salt stress; still, this has been shown, for example, in Ligustrum vulgare (Agati et al., 2011) or in two salt-tolerant Juncus species, J. maritimus and $J$. acutus - but not in the salt-sensitive congener $J$. articulatus (Al Hassan et al., 2017).

\section{Ecological relevance of flavonoids in abiotic stress tolerance mechanisms}

The strong antioxidant activity of phenolic compounds, especially that of many flavonoids, has been demonstrated in many in vitro assays; it is clear that they are able to block the accumulation of ROS to excessive levels and to eliminate ROS once they are formed (Pollastri and Tattini, 2011; Bose et al., 2013). However, experimental data supporting a biologically relevant role of these compounds in the natural habitats of the plants are not so abundant. Most available information refers to the model Arabidopsis thaliana and a few crop species, which are all rather sensitive to stress, and to experiments performed under controlled but artificial - conditions in the greenhouse; field data on stress tolerant wild species are still very scarce. In addition, considering the disparate biological functions of flavonoids, their synthesis can be activated by different external and internal signals unrelated to abiotic stress, which may mask their specific role in stress tolerance mechanisms. Nevertheless, this functional role is supported by some of the studies mentioned in the previous section, reporting flavonoid accumulation in particular species subjected to abiotic stress in the field. These results have been recently generalised and strengthen by an extensive field study, which included a large number of wild species of several families growing in diverse natural habitats; leaf material was collected from all the plants in three successive seasons, therefore under a wide range of environmental conditions, and the levels of total phenolic compounds and antioxidant flavonoids were determined (Bautista et al., 2016). Despite the aforementioned limitations, and quantitative differences observed among different taxa, it was possible to establish statistically significant correlations between phenolics and flavonoid contents and the intensity of distinct environmental stresses. Specifically, flavonoid levels showed a strong positive correlation with altitude (that is, with UV radiation intensity), and correlated also with environmental parameters associated with drought stress: positively with water deficit, evapotranspiration and temperature, and negatively with soil moisture and accumulated rainfall. 


\section{Conclusions}

Flavonoids constitute an extremely complex group of plant phenolic compounds with a wide range of biological functions. There is strong evidence for their relevant role in the mechanisms of defence against abiotic stresses, including drought and salinity, mediated by their capacity to scavenge 'reactive oxygen species' (ROS) generated under stress conditions. The strong antioxidant activity of many of these secondary metabolites, responsible for this specific role in plants, appears to be also the reason for their alleged (or, in some cases, demonstrated) beneficial effects for human health, by including flavonoid-rich fruits and vegetables in the diet, using them as food supplements or nutraceuticals, and also for the possible development of drugs with diverse pharmacological activities. Flavonoids represent therefore a unique example of biochemicals of interest for researchers working in diverse scientific fields: plant biology and ecology, phytochemistry, human nutrition, biomedicine and pharmacology.

\section{References}

Agati G, Biricolti S, Guidi L, Ferrini F, Fini A, Tattini M (2011). The biosynthesis of flavonoids is enhanced similarly by UV radiation and root zone salinity in L. vulgare leaves. Journal of Plant Physiology $168: 204212$.

Agati G, Brunetti C, Di Ferdinando M, Ferrini F, Pollastri S, Tattini M (2013). Functional roles of flavonoids in photoprotection: New evidence, lessons from the past. Plant Physiology and Biochemistry 72:35-45.

Al Hassan M, Chaura J, Donat-Torres MP, Boscaiu M, Vicente O (2017). Antioxidant responses under salinity and drought inthree closely related wild monocots with different ecological optima. AoB PLANTS 9: plx009.doi:10.1093/aobpla/plx009.

Apel K, Hirt H(2004). Reactive oxygen species: metabolism, oxidative stress, and signal transduction. Annual Review of Plant Biology 55:373-399.

Athar M, BackJH, Tang X, Kim KH, Kopelovich L, Bickers DR, Kim AL (2007). Resveratrol: a review of pre-clinical studies for human cancer prevention. Toxicology and Applied Pharmacology 224:274283.

Bachereau F, Marigo G, Asta J (1998). Effect of solar radiation (UV and visible) at high altitude on CAM-cycling and phenolic compounds biosynthesis in Sedum album. Physiologia Plantarum 104:203-210.

Baldasquin-Caceres B, Gomez-Garcia FJ, López-Jornet P, Castillo-SanchezJ, Vicente-Ortega V (2014). Chemopreventive potential of phenolic compounds in oral carcinogenesis. Archives of Oral Biology 59(10):1101-1107.

Ballizany WL, Hofmann RV, Jahufer MZZ, Barrett BB (2012). Multivariate associations of flavonoid and biomass accumulation in white clover (Trifolium repens) under drought. Functional Plant Biology 39:167-177.

BaurJA, Sinclair DA (2006). Therapeutic potential of resveratrol: the in vivo evidence. Nature Reviews Drug Discovery 5(6):493-506.

Bieza K, Lois R (2001). An Arabidopsis mutant tolerant to lethal ultraviolet$\mathrm{B}$ levels shows constitutively elevated accumulation of flavonoids and other phenolics. Plant Physiology 126:1105-1115.
Blumthaler M, Ambach M, Ellinger R (1997).Increase in solar UV radiation with altitude. Journal of Photochemistry and Photobiology B 39:130134.

Bose J, Rodrigo-Moreno A, Shabala S (2013). ROS homeostasis in halophytes in the context of salinity stress tolerance. Journal of Experimental Botany 65:1241-1257.

Brown DE, Rashotte AM, Murphy AS, NormanlyJ, Tague BW, Peer WA, Taiz L, Muday GK (2001). Flavonoids act as a negative regulators of auxin transport in vivo in Arabidopsis. Plant Physiology 126:524535.

Burchard P, Bilger W, Weissenböck G (2000). Contribution of hydroxycinnamates and flavonoids to epidermal shielding of UV-A and UV-B radiation in developing rye primary leaves as assessed by ultraviolet-induced chlorophyll fluorescence measurements. Plant Cell and Environment 23:1373-1380.

Butelli E, Titta L, Giorgio M, Mock HP, Matros A, Peterek S, ... Martin C (2008). Enrichment of tomato fruit with health-promoting anthocyanins by expression of select transcription factors. Nature Biotechnology 26:1301-1308.

Cheynier V, Comte G, Davies KM, Lattanzio V, Martens S (2013). Plant phenolics: recent advances on their biosynthesis, genetics, and ecophysiology. Plant Physiology and Biochemistry 72:1-20.

Coman C, Rugina OD, Socaciu C (2012). Plants and natural compounds with antidiabetic action. Notulae Botanicae Horti Agrobotanici ClujNapoca 40:314-325.

Di Ferdinando M, Brunetti C, Fini A, Tattini M (2012). Flavonoids as antioxidants in plants under abiotic stresses. In: Ahmad P, Prasad MNV (Eds). Abiotic stress responses in plants: metabolism, productivity and sustainability.Springer, New Yorkpp 159-179.

Downie JA (2014). Legume nodulation. Current Biology 24(5):R184 R190.

Fini A, Brunetti C, Di Ferdinando M, Ferrini F, Tattini M (2011). Stressinduced flavonoid biosynthesis and the antioxidant machinery of plants. Plant Signaling and Behaviour 6:709-711.

Fita A, Rodríguez-Burruezo A, Boscaiu M, Prohens J, Vicente O (2015). Breeding and domesticating crops adapted to drought and salinity: a new paradigm for increasing food production. Frontiers in Plant Science 6,978. doi:10.3389/fpls.2015.00978.

Gould KS, Lister C (2006). Flavonoid function in plants. In: Andersen ØM, Marham KR (Eds). Flavonoids, chemistry, biochemistry and application. CRC Press, Boca Raton, FL pp 397-442.

Guarente L, Picard F (2005). Calorie restriction - the SIR2 connection. Cell 120(4):473-482

Halliwell B (2006). Reactive species and antioxidants. Redox biology is a fundamental theme of aerobic life. Plant Physiology 141:312-322

Harborne JB, Williams C (2000). Advances in flavonoid research since 1992. Phytochemistry 55:481-504

Hernández I, Alegre L, Munné-Bosch S (2004). Drought-induced changes in flavonoids and other low molecular weight antioxidants in Cistus clusii grown under Mediterranean field conditions. Tree Physiology 24:13031311.

Howitz KT, Bitterman KJ, Cohen HY, Lamming DW, Lavu S, WoodJG, ... Sinclair DA (2003). Small molecule activators of sirtuins extend Saccharomyces cerevisiae lifespan. Nature 425(6954):191-196. 
20

Jaakola L, Määttä-Riihinen K, Kärenlampi S, Hohtola A (2004). Activation of flavonoid biosynthesis by solar radiation in bilberry (Vaccinium myrtillus L.) leaves. Planta 218:721-728.

Jang M, Cai L, Udeani GO, Slowing KV, Thomas CF, Beecher CW, ... Pezzuto JM (1997). Cancer chemopreventive activity of resveratrol, a natural product derived from grapes. Science 275(5297):218-220.

Keys A., Grande F (1957). Role of dietary fat in human nutrition. III. Diet and the epidemiology of coronary heart disease. American Journal of Public Health and the Nation's Health 47:1520-1530.

Keys A (1970). Coronary heart disease in seven countries. Circulation 41 (4S1):1-98.

Kirakosyan A, Seymour E, Kaufman PB, Warber S, Bolling S, Chang SC (2003). Antioxidant capacity of polyphenolic extracts from leaves of Crataegus laevigata and Crataegus monogma (Hawthorn) subjected to drought and cold stress. Journal of Agricultural and Food Chemistry 51:3973-3976.

Koes RE, Spelt CE, Mol JNM (1989). The chalcone synthase multigene family of Petunia hybrida (V30): differential, light regulated expression during flower development and UV light induction. Plant Molecular Biology 12:213-225.

Kopp P (1998). Resveratrol, a phytoestrogen found in red wine. A possible explanation for the conundrum of the 'French paradox'? European Journal of Endocrinology 138(6):619-620.

Koubová J, Guarente L (2003). How does calorie restriction work? Genes \& Development 17(3):313-321.

Kumar S, Pandey AK (2013). Chemistry and biological activities of flavonoids: an overview. The Scientific World Journal 2013:1-16.

Lavola A (1998). Accumulation of flavonoids and related compounds in birch induced by UV-Birradiance. Tree Physiology 18:53-58.

Li J, Ou-Lee TM, Raba R, Amundson RG, Last RL (1993). Arabidopsis flavonoid mutants are hypersensitive to UV-B radiation. Plant Cell 5:171-179.

Martorell P, Forment JV, de Llanos R, Montón F, Llopis S, González N, ... Ramón D (2011). Use of Saccharomyces cerevisiae and Caenorhabditis elegans as model organisms to study the effect of cocoa polyphenols in the resistance to oxidative stress. Journal of Agricultural and Food Chemistry 59(5):2077-2085.

Mazzucato A, Willems D, Bernini R, Picarella ME, Santangelo E, Ruiu F, ... Soressi GP (2013). Novel phenotypes related to the breeding of purplefruited tomatoes and effect of peel extracts on human cancer cell proliferation. Plant Physiology and Biochemistry 272:125-133.

Middleton E Jr, Kandaswami C, Theoharides TC (2000). The effects of plant flavonoids on mammalian cells: implications for inflammation, heart disease and cancer. Pharmacological Reviews 52:673-751.

Murai Y, Takemura S, Takeda K, Kitajima K, Iwashina T (2009). Altitudinal variation of $U V$-absorbing compounds in Plantago asiatica. Biochemical Systematics and Ecology 37:378-384.

Nakabayashi R, Yonekura-Sakakibara K, Urano K, Suzuki M, Yamada Y, Nishizawa T, ... Saito K (2014). Enhancement of oxidative and drought tolerance in Arabidopsis by overaccumulation of antioxidant flavonoids. Plant Journal 77:367-379.

Napoli CA, Fahy D, Wang HY, Taylor LP (1999). white anther: A petunia mutant that abolishes pollen flavonoid accumulation, induces male sterility, and is complemented by a chalcone synthase transgene. Plant Physiology 120:615-622.

Pacurar DI, Thordal-Christensen H, Pacurar M, Pamfil D, Botez C, Bellini C (2011). Agrobacterium tumefaciens: From crown gall tumors to genetic transformation. Physiological and Molecular Plant Pathology 76:76-81.

Pękal A, Pyrzynska K (2014). Evaluation of aluminium complexation reaction for flavonoid content assay. Food Analytical Methods 7:17761782.

PollastriS, Tattini M(2011). Flavonols: old compounds for old roles. Annals ofBotany 108:1225-1233.

Ravishankar D, Rajora AK, Greco F, Osborn HM (2013). Flavonoids as prospective compounds for anti-cancer therapy. The International Journal of Biochemistry \& Cell Biology 45:2821-2831.

Rice-Evans CA, Miller NJ, Paganga G (1996). Structure-antioxidant activity relationships of flavonoids and phenolic acids. Free Radical Biology \& Medicine 20:933-956.

Rieger G, Müller M, Guttenberger H, Bucar F (2008). Influence of altitudinal variation on the content of phenolic compounds in wild populations of Calluna vulgaris, Sambucus nigra, and Vaccinium myrtillus. Journal of Agricultural and Food Chemistry 58:9080-9086.

Romano B, Pagano E, Montanaro V, Fortunato AL, Milic N, Borrelli F (2013). Novel insights into the pharmacology of flavonoids. Phytotherapy Research 27:1588-1596.

Rozema J, Bjorn LO, Bornman JF, Gaberscik A, Häder DP, Trost T, ... Meijkamp BB (2002). The role of UV-B radiation in aquatic and terrestrial ecosystems - an experimental and functional analysis of the evolution of UV-absorbing compounds. Journal of Photochemistry and Photobiology B: Biology 66:2-12.

Rozema J, van de Staaij J, Björn LO, Caldwell MM (1997). UV-B as an environmental factor in plant life: stress and regulation. Trends in Ecology \& Evolution 12:22-28.

Schulze-Lefert P, Dangl JL, Becker-André M, Hahlbrock K, Schulz W (1989). Inducible in vivo DNA footprints define sequences necessary for UV light activation of the parsley chalcone synthase gene. EMBO Journal 8:651-656.

Siemann E, Creasy L (1992). Concentration of the phytoalexin resveratrol in wine. American Journal of Enology and Viticulture 43:49-52.

Spitaler R, Winkler A, Lins I, Yanar S, Stuppner H, Zidorn C (2008). Altitudinal variation of phenolic contents in flowering heads of Arnica montana cv. ARBO: a 3-year comparison. Journal of Chemical Ecology 34:369-375.

St Leger AS, Cochrane AL, Moore F (1979). Factors associated with cardiac mortality in developed countries with particular reference to the consumption of wine. Lancet 1:1017-1020.

Stapleton AE, Walbot V(1994). Flavonoids can protect maize DNA from the induction of UV radiation damage. Plant Physiology 105:881-889.

Takahashi M, Asada K (1988). Superoxide production in aprotic interior of chloroplast thilakoids. Archives of Biochemistry and Biophysics 267:714722

Tattini M, Galardi C, Pinelli P, Massai R, Remorini D, Agati G (2004). Differential accumulation of flavonoids and hydroxycinnamates in leaves of Ligustrum vulgare under excess light and drought stress. New 
Phytologist 163:547-561.

Taylor LP, Jorgensen R (1992). Conditional male fertility in chalcone synthase-deficient petunia. Journal of Heredity 83:11-17.

Treutter D (2005). Significance of flavonoids in plant resistance and enhancement of their biosynthesis. Plant Biology 7:581-591.

Treutter D (2006). Significance of flavonoids in plant resistance: a review. Environmental Chemistry Letters 4:147-157.

Valenzano DR, Terzibasi E, Genade T, Cattaneo A, Domenici L, Cellerino A (2006). Resveratrol prolongs lifespan and retards the onset of agerelated markers in a short-lived vertebrate. Current Biology 16(3):296300.

Vidavalur R, Otani H,Singal PK, MaulikN (2006). Significance of wine and resveratrol in cardiovascular disease: French paradox revisited. Experimental and Clinical Cardiology 11(3):217-225.

Williams CA, Grayer RJ (2004). Anthocyanins and other flavonoids. Natural Products Reports 21:539-573.

Winkel-Shirley B (2002). Biosynthesis of flavonoids and effect of stress. Current Opinion in Plant Biology 5:218-223.
Wood JG, Rogina B, Lavu S, Howitz K, Helfand SL, Tatar M, Sinclair D (2004). Sirtuin activators mimic caloric restriction and delay ageing in metazoans. Nature 430:686-689.

Yerushalmy J, Hilleboe HE (1957) Fat in the diet and mortality from heart disease. A methodologic note. New York State Journal of Medicine 57:2343-2354.

Ylstra B, Touraev A, Benito Moreno RM, Stöger E, van Tunen AA, Vicente O, ... Heberle-Bors E (1992). Flavonols stimulate development, germination and tube growth of tobacco pollen. Plant Physiology 100:902-907.

Zhishen J, Mengcheng T, Jianming W (1999). The determination of flavonoid contents in mulberry and their scavenging effects on superoxide radicals. Food Chemistry 64:555-559.

Zidorn C, Schubert B, Stuppner H (2005). Altitudinal differences in the contents of phenolics in flowering heads of three members of the tribe Lactuceae (Asteraceae) occurring as introduced species in New Zealand. Biochemical Systematics and Ecology 33:855-872. 